\title{
Estudo epidemiológico da doença de Cushing no Hospital Universitário Walter Cantídio
}

\section{Epidemiological study of Cushing disease in Walter Cantídio University Hospital}

\author{
Ivana da Ponte Meloํ. Ana Rosa Pinto Quidute².
}

1 Universidade Federal do Ceará (UFC), Hospital Geral do Ceará (HGF), Fortaleza, Ceará, Brasil. 2 Hospital Universitário Walter Cantídio (HUWC), Universidade Federal do Ceará (UFC), Núcleo de Pesquisa e Desenvolvimento de Medicamentos da UFC (NPDM - UFC), Fortaleza, Ceará, Brasil.

\section{RESUMO}

Objetivos: avaliar a epidemiologia dos pacientes com doença de Cushing que são acompanhados no Hospital Universitário Walter Cantídio (HUWC) e confrontar nossos dados com o da literatura atual. Metodologia: foi realizado um estudo observacional, longitudinal e retrospectivo sendo incluídos os pacientes com acompanhamento atual ou prévio por doença de Cushing (DC) no serviço de endocrinologia do HUWC. A análise dos dados foi realizada pelo programa SPSS $^{\circledR}$ e o nível de significância adotado para os testes estatísticos foi de 5\% ( $<<0,05)$. O projeto foi submetido, por meio da Plataforma Brasil, ao Comitê de Ética e Pesquisa do HUWC. Resultados: observou-se uma maior prevalência de lesões menores que $1 \mathrm{~cm}$. A maioria dos pacientes realizou cirurgia transesfenoidal como terapia de primeira linha. O controle nos primeiros 6 meses de doença foi obtido em $71,42 \%$ dos pacientes que realizaram algum tipo de cirurgia, porém, com importante índice de recidiva. Conclusões: no presente estudo, foi observada uma alta incidência de recidiva após o tratamento de primeira linha. Devido à alta morbidade e a resposta terapêutica, nem sempre desejada nos tratamentos de primeira linha, a DC é ainda bastante desafiadora para o endocrinologista. É importante o seguimento desses pacientes a longo prazo.

Palavras-chave: Hipersecreção hipofisária de ACTH. Recidiva. Terapêutica.

\section{ABSTRACT}

Objectives: To evaluate the epidemiology of patients with Cushing's disease who are followed at the Hospital Universitário Walter Cantídio (HUWC) and compare our data with that of the current literature. Methods: An observational, longitudinal and retrospective study was carried out, including patients with current or previous Cushing's Disease (DC) follow-up at the HUWC Endocrinology Service. Data analysis was performed by the SPSS program, and the significance level adopted for the statistical tests was $5 \%(\mathrm{p}<0.05)$. The project was submitted, through the Brazil Platform, to the Ethics and Research Committee of the HUWC. Results: A higher prevalence of lesions smaller than $1 \mathrm{~cm}$ was observed. Most patients underwent transsphenoidal surgery as first-line therapy. The control in the first 6 months of disease was obtained in $71.42 \%$ of the patients who performed some type of surgery, but with an important relapse rate. Conclusions: In the present study, a high incidence of recurrence was observed after first-line treatment. Due to the high morbidity and therapeutic response, not always desired in first-line treatments, DC is still quite challenging for the endocrinologist. Long-term follow-up of these patients is important.

Keywords: Pituitary ACTH Hypersecretion. Recurrence. Therapeutics.

Autor correspondente: Ivana da Ponte Melo, Rua Carlos Vasconcelos, 1946, Aldeota, Fortaleza, Ceará. CEP: 60115-171. Telefone: +55 85 998103840. E-mail: ivanapmelo@yahoo.com.br

Conflito de interesses: Não há qualquer conflito de interesses por parte de qualquer um dos autores.

Recebido em: 29 Jan 2019; Revisado em: 30 Out 2019; Aceito em: 14 Nov 2019. 


\section{INTRODUÇÃO}

A Síndrome de Cushing é um estado clínico caracterizado pela exposição prolongada de níveis inapropriadamente aumentados de glicocorticoides e é associada a significativa morbidade e mortalidade. ${ }^{1} \mathrm{O}$ aumento exógeno através do uso de doses suprafisiológicas de glicorticoide é a causa mais comum da Síndrome de Cushing. No entanto, a doença de Cushing (DC), tumor pituitário secretor de corticotropina, é a causa mais comum de Cushing endógena. ${ }^{2}$ Apesar dos estudos populacionais mostrarem uma baixa incidência da síndrome de Cushing endógena, a avaliação de pacientes com diabetes e hipertensão mal controlados demonstrou que essa doença é subdiagnosticada. ${ }^{3-6}$ A Cirurgia Transesfenoidal (CTE) é considerada o tratamento inicial para essa entidade. Porém, se sabe que a recorrência pode chegar a aproximadamente 25 a $50 \%$ em pacientes seguidos por 5 a 10 anos em centros terciários. ${ }^{7,8} \mathrm{O}$ Hospital Universitário Walter Cantídio (HUWC) é um dos centros terciários responsável pelo acompanhamento desses pacientes no estado do Ceará. Dessa forma, é de grande importância avaliar o índice de cura com o tratamento de primeira linha, além dos fatores que podem estar associados a uma melhor resposta nos pacientes acompanhados nesse serviço. Além disso, tendo em vista o alto índice de recorrência descrito na literatura, também se faz importante ver a resposta às terapias de segunda linha. O objetivo geral do presente estudo é avaliar a epidemiologia dos pacientes com doença de Cushing que são acompanhados no HUWC e confrontar nossos dados com o da literatura vigente no momento. Como objetivos específicos pretendemos observar a resposta ao tratamento de primeira linha (CTE).

\section{MATERIAIS E MÉTODOS}

Foi realizado um estudo observacional, longitudinal e retrospectivo.

Nesse estudo, estão inclusos todos os pacientes com acompanhamento atual ou prévio por doença de Cushing no serviço de endocrinologia e diabetes do HUWC, no período de 1980 a 2018. Foram obtidos os dados contidos nos prontuários dessa instituição referente a cada paciente analisado pelo estudo. Para o levantamento de quais dados eram mais importantes para serem coletados, foi realizada revisão da literatura nacional e internacional através das bases de dados online Medline, Scielo e ScienceDirect, utilizando para pesquisa os termos: doença de Cushing, diagnóstico, recidiva e tratamento. Foram incluídos artigos em português e em inglês. Os dados coletados são referentes ao tamanho da lesão, idade ao diagnóstico, exame utilizado para fornecer o diagnóstico, tratamento de primeira linha, tratamento das recidivas e seus efeitos adversos. Não foi necessária nenhuma forma de contato direto com o paciente para a coleta das informações. O sigilo, que assegura a privacidade e o anonimato dos sujeitos quanto aos dados confidenciais envolvidos na pesquisa, foi garantido.

A análise dos dados se deu através do programa SPSS ${ }^{\circledR}$. Devido ao tamanho reduzido da amostra e à falta de dados em alguns prontuários, foi utilizado o Teste Exato de Fisher para avaliação de significância estatística. $\mathrm{O}$ nível de significância adotado para o teste foi de $5 \%(\mathrm{p}<0,05)$. O projeto foi submetido e aprovado, por meio da Plataforma Brasil, ao Comitê de Ética e Pesquisa do Hospital Universitário Walter Cantídio. A pesquisa foi realizada de acordo com os critérios estabelecidos pela Declaração de Helsinki com as suas modificações (Bull World Health Organ 2001; 79:373-374).

\section{RESULTADOS}

Foram incluídos 39 pacientes com diagnóstico de doença de Cushing no período analisado. A média de idade dos pacientes foi de 29,48 anos $\pm 9,139$ (variação entre 14 e 51 anos), sendo o caso mais jovem um indivíduo de 14 anos do sexo feminino. O tempo entre o surgimento dos sintomas e a admissão no serviço foi em média de 3,11 anos $\pm 3,62$ (variação entre 0,33 e 15 anos). As lesões hipofisárias foram divididas em microadenomas, quando menores do que $1 \mathrm{~cm}$, ou macroadenomas quando maiores que $1 \mathrm{~cm}$. No presente estudo, foi observada uma maior prevalência de microadenomas, responsáveis por $43,5 \%$ dos casos. Os macroadenomas corresponderam à $35,8 \%$ dos casos, e faltou informação a respeito do tamanho da lesão em 20,5\% dos casos. Em relação à terapia de primeira linha, a maioria dos pacientes foram submetidos a cirurgia transesfenoidal, totalizando 33 pacientes que realizaram esse procedimento $(84,61 \%$ dos pacientes) enquanto apenas $2(5,12 \%)$ realizaram cirurgia transcraniana.

Dos 35 que foram submetidos a cirurgia, em $71,42 \%$ foi possível avaliar o controle da doença tanto clínico quanto bioquímico (através da realização de um dos seguintes exames: teste de supressão com $1 \mathrm{mg}$ de dexametasona, cortisol livre urinário em 24 horas ou cortisol salivar às 23 horas) dentro dos primeiros seis meses.

Em relação às taxas de recorrência/recidiva, só havia essa informação em 24 dos 39 prontuários analisados. Desses 24 casos, 54,16\% apresentaram recorrência/recidiva avaliadas em média 90 dias após o procedimento cirúrgico. Do restante dos indivíduos analisados no período até a presente revisão, $4,16 \%$ está em investigação de recidiva, 4,16\% perdeu o seguimento no serviço e $37,5 \%$ não apresentaram nova

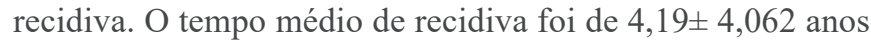
(variação entre 0,75 e 15 anos). O controle da doença nos primeiros 6 meses após a cirurgia foi observado em 87,5\% dos microadenomas e em $61,5 \%$ dos macroadenomas. Porém, utilizando o teste exato de Fisher, não observamos associação estatística entre tamanho da lesão macro ou micro adenoma e a taxa de controle da doença nos primeiros 6 meses $(p=$ 0,192).

\section{DISCUSSÃO E REVISÃO DA LITERATURA}

A Doença de Cushing é causada mais frequentemente por crescimento monoclonal lento e benigno de microadenomas (menor que $10 \mathrm{~mm}){ }^{9}$ 
$\mathrm{O}$ adenoma hipofisário secreta grande quantidade de ACTH levando a um aumento na produção de cortisol e a uma supressão endógena na secreção de CRH e ACTH. O ACTH e cortisol plasmático perdem seu ciclo circadiano. Eles ficam resistentes ao estímulo fisiológico e não respondem adequadamente a alça de feedback negativa. ${ }^{10}$

Essa doença ainda é um grande desafio diagnóstico, já que além de ser rara, a sua apresentação clínica pode ser confundida com de outras condições mais prevalentes como diabetes melito tipo 2, osteoporose e hipertensão arterial sistêmica. Além disso, os critérios que levam ao seu diagnóstico são complexos e desconhecidos por muitos clínicos. Isso tudo levanta o questionamento se a Doença de Cushing estaria sendo subdiagnosticada.

No presente estudo foi observada uma média de idade ao diagnóstico de 29,48 \pm 9,139 anos. Essa informação diverge de outras revisões da literatura mundial que demonstra uma idade média de 41,4 anos ao diagnóstico. ${ }^{11}$ Os dados epidemiológicos do HUWC demonstraram um tempo médio de 3,11 \pm 3,62 anos entre o início dos sintomas até à admissão no serviço de endocrinologia. Tal informação é preocupante, já que existem dados que demonstram que o risco de morte por síndrome de Cushing foi maior durante o primeiro ano após a admissão inicial e um número de mortes ocorreu antes que um tratamento específico pudesse ser realizado. ${ }^{12}$

Nossa casuística demonstrou uma taxa de remissão

\section{REFERÊNCIAS}

1. Newell-Price J, Bertagna X, Grossman AB, Nieman LK. Cushing's syndrome. Lancet. 2006;367(9522):1605-17.

2. Isidori AM, Kaltsas GA, Pozza C, Frajese V, Newell-Price J, Reznek RH, et al. The ectopic adrenocorticotropin syndrome: clinical features, diagnosis, management, and long-term follow-up. J Clin Endocrinol Metab. 2006;91(2):371-7.

3. Leibowitz G, Tsur A, Chayen SD, Salameh M, Raz I, Cerasi E, et al. Pre-clinical Cushing's syndrome: an unexpected frequent cause of poor glycaemic control in obese diabetic patients. Clin Endocrinol (Oxf). 1996;44(6):717-22.

4. Catargi B, Rigalleau V, Poussin A, Ronci-Craix N, Bex V, Vergnot $\mathrm{V}$, et al. Occult Cushing's syndrome in type-2 diabetes. J Clin Endocrinol Metab. 2003;88(12):5808-13.

5. Omura M, Saito J, Yamaguchi K, Kakuta Y, Nishikawa T. Prospective study on the prevalence of secondary hypertension among hypertensive patients visiting a general outpatient clinic in Japan. Hypertens Res. 2004;27(3):193-202.

6. Terzolo M, Reimondo G, Chiodini I, Castello R, Giordano $\mathrm{R}$, Ciccarelli E, et al. Screening of Cushing's syndrome in outpatients with type 2 diabetes: results of a prospective multicentric study in Italy. J Clin Endocrinol Metab. 2012;97(10):3467-75.

7. Patil CG, Prevedello DM, Lad SP, Vance ML, Thorner MO, Katznelson L, et al. Late recurrences of Cushing's disease after bioquímica de $71,42 \%$. Esse dado é semelhante ao de uma revisão sistemática, que incluiu 8113 pacientes com doença de Cushing tratados com cirurgia transesfenoidal, que apresentou taxa de remissão bioquímica após CTE de 76\% (95\% IC 72 a $79 \%$ ). ${ }^{13}$ Porém, nesse mesmo estudo, foi observada uma taxa de recorrência a longo prazo de $10 \%$, sendo bem menor do que os 54,16\% encontrados no nosso serviço. O sucesso da cirurgia é dependente de fatores como experiência do cirurgião e localização do tumor. ${ }^{14}$ Além disso, ainda hoje, existe uma heterogeneidade nos critérios laboratoriais que definem remissão e recorrência da DC. Os testes laboratoriais mais comumente utilizados são o cortisol basal da manhã, cortisol livre urinário ou a combinação desses dois testes. ${ }^{13}$ As recidivas geralmente ocorrem dentro de cinco anos após a cirurgia, mas podem acontecer após 16 anos ou mais. ${ }^{15}$ Isso foi semelhante ao desfecho desse serviço, no qual a média da recidiva foi de 4,19 anos, com o tempo máximo de recidiva de 15 anos.

Devido às altas morbidade e mortalidade, a doença de Cushing constitui um grande desafio para o endocrinologista. A cirurgia transfenoidal ainda é o tratamento de primeira linha, porém, essa terapêutica pode ser seguida por recidiva em muitos casos. Altas taxas de remissão após a cirurgia são observadas em adenomas pequenos e com histologia do tumor positiva para ACTH. ${ }^{13}$ Dessa forma, pacientes com DC precisam ser acompanhados por um período indefinido após a CTE.

initial successful transsphenoidal surgery. J Clin Endocrinol Metab. 2008;93(2):358-62.

8. Atkinson AB, Kennedy A, Wiggam MI, McCance DR, Sheridan B. Long-term remission rates after pituitary surgery for Cushing's disease: the need for long-term surveillance. Clin Endocrinol (Oxf). 2005;63:549-59.

9. Leibowitz G, Tsur A, Chayen SD, Salameh M, Raz I, Cerasi E, et al. Pre-clinical Cushing's syndrome: an unexpected frequent cause of poor glycaemic control in obese diabetic patients. Clin Endocrinol (Oxf). 1996;44(6):717-22.

10. Catargi B, Rigalleau V, Poussin A, Ronci-Craix N, Bex V, Vergnot V, et al. Occult Cushing's syndrome in type-2 diabetes. J Clin Endocrinol Metab. 2003;88(12):5808-13.

11. Steffensen C, Bak AM, Rubeck KZ, Jorgensen JO. Epidemiology of Cushing's syndrome. Neuroendocrinology. 2010;92(suppl1):1-5.

12. Lindholm J, Juul S, Jorgensen JO, Astrup J, Bjerre P, FeldtRasmussen U, et al. Incidence and late prognosis of Cushing's syndrome: a population-based study. J Clin Endocrinol Metab. 2001;86(1):117-23.

13. Abu Dabrh AM, Singh Ospina NM, Al Nofal A, Farah WH, Barrionuevo P, Sarigianni M, et al. Predictors of biochemical remission and recurrence after surgical and radiation treatments of Cushing disease: a systemic review and meta-analysis. Endocr Pract. 2016;22(4):466-75. 
14. Fleseriu M, Hamrahian AH, Hoffman AR, Kelly DF, Katznelson L. AACE Neuroendocrine and Pituitary Scientific Committee. Diagnosing recurrent Cushing disease. Endocr Pract. 2016;22(12):1436-48.
15. Pivonello R, De Leo M, Cozzolino A, Colao A. The treatment of Cushing's disease. Endocr Rev. 2015;36(4):385-486.

\section{Como citar:}

Melo IP, Quidute AR. Estudo epidemiológico da doença de Cushing no Hospital Universitário Walter Cantídio. Rev Med UFC. 2020 julset;60(3):15-18 